\title{
The ERS Research Agency: the beginning
}

\author{
Joan B. Soriano ${ }^{1}$, James Paton ${ }^{2}$, Fernando Martin Burrieza ${ }^{3}$, Werner Bill ${ }^{3}$, \\ Carine Pannetier ${ }^{4}$, Stefano Aliberti ${ }^{5}$, lan M. Adcock ${ }^{6}$, Scott Wagers ${ }^{7}$ and \\ Giovanni Battista Migliori ${ }^{8}$
}

Affiliations: ${ }^{1}$ Instituto de Investigación Hospital Universitario de la Princesa (IISP), Universidad Autónoma de Madrid, Cátedra UAM-Linde, Madrid, Spain. ${ }^{2}$ School of Medicine, University of Glasgow, Glasgow, UK. ${ }^{3}$ ERS Executive Office, European Respiratory Society, Lausanne, Switzerland. ${ }^{4}$ ERS Science and Education, European Respiratory Society, Lausanne, Switzerland. ${ }^{5}$ School of Medicine and Surgery, University of Milan Bicocca, Milano, Italy. ${ }^{6}$ Cell and Molecular Biology, Airways Disease Section, National Heart and Lung Institute Faculty of Medicine, Imperial College London, London, UK. ${ }^{7} \mathrm{BioSci}$ Consulting, Maasmechelen, Belgium. ${ }^{8} \mathrm{WHO}$ Collaborating Centre for TB and Lung Diseases, Fondazione S. Maugeri, Care and Research Institute, Tradate, Italy.

Correspondence: Giovanni Battista Migliori, WHO Collaborating Centre for TB and Lung Diseases, Fondazione S. Maugeri, Care and Research Institute, 21049 Tradate, Italy. E-mail: giovannibattista.miglioriafsm.it

@ERSpublications

As part of its 2013-2018 strategic plan, the European Respiratory Society launches the ERS Research Agency http://ow.ly/WZyac

\section{Thriving in a challenging research environment}

The modern research environment is much more complex and challenging than before [1]. Mechanistic research has become increasingly sophisticated and specialised, while new clinical research approaches have vastly increased the amount of data generated. These new developments bring a number of challenges.

The first challenge is collecting and analysing the current deluge of data. This requires more than just one laboratory group, one institution or one national institute. Today's research requires the coordination of wide ranging expertise that is often only available on the international or even global level. A second challenge involves the increased availability of patient data, which has raised concerns about privacy of personal data resulting in new legal and regulatory constraints for handling personal data in a multinational and multicultural environment, making large-scale clinical research even more complicated. A third challenge is that, currently, the greatest economic burden in healthcare comes from chronic, noncommunicable disease [2]. Natural history studies requiring timescales measured in decades are needed if this burden is to be reduced. However, natural history studies are not only difficult to conduct, but they are also challenging because most research funding is for time periods of 3-5 years while studies of 10 years or longer are needed. Finally, a fourth challenge is difficulty in obtaining funding for respiratory research. Obtaining funding at a European level has always been difficult because of competition from other areas and because of the complex policies and procedures. The Horizon 2020 programme represents a great opportunity for increasing respiratory research, but the competition appears to be ever fiercer. There is also a perception that on a national level all research funding is becoming ever more difficult to obtain $[3,4]$.

Thus, today's translational researchers face many challenges, including more difficult and complex European regulations [5], and have a number of unfulfilled needs. In order to help researchers meet these needs, medical societies such as the European Respiratory Society (ERS) could assist to coordinate multinational research efforts [1].

In 2013, the ERS Leadership formulated a strategy for the following 5 years that aimed to serve ERS members better and further support the overall mission of the ERS, taking into account changes occurring both within the ERS and within the respiratory field. The fourth of the eight agreed decisions,

Received: Dec 142015 | Accepted: Dec 162015

Conflict of interest: F. Martin Burrieza, W. Bill and C. Pannetier are employees of the European Respiratory Society. Other disclosures can be found alongside the online version of this article at erj.ersjournals.com

Copyright @ERS 2016 
"Strengthening Science”, included an action to: “... Develop a plan to launch an ERS Research Agency, which will coordinate and fund respiratory research across Europe" [6].

A team composed of ERS officers, staff and external experts began to define a plan to move forward with the development of a Research Agency and to assist the ERS Leadership in defining the opportunities and implications of launching this project (figure 1). This group's work culminated in an internal white paper that comprised two main sections: a summary of inputs from ERS leadership, officers and ERS affiliated organisations about the roles, possible benefits and risks of a Research Agency; and a portfolio of projected activities, structure and initial steps for establishing a Research Agency. This editorial summarises the white paper and aims to describe proposed activities, a structure, and a financial model for the ERS Research Agency.

\section{Central aim}

The central aim of the Research Agency is to facilitate respiratory research through the coordination and support of the respiratory research community, and to assist in its efforts to obtain funding. The intent is not to develop a new funding organisation that provides grants for specific projects, but to adopt a self-financing business model, which is expected to diversify the income sources of the ERS in the medium term.

\section{Portfolio of activities and structure}

A number of core concepts were used to guide the development of a projected portfolio of activities within the Research Agency. These concepts were mainly based on feedback from the membership, experience within the core team, discussion with ERS Assembly Heads and principles gleaned from the study of other existing research organisations. The activities and structure chosen within the Research Agency should be established according to the following principles: 1) start from the existing and successful ERS portfolio of both scientific and educational activities (guidelines, education and fellowships); 2) adopt an incremental approach that constantly includes opinions and representatives of ERS assemblies in the inception and development of any project; 3 ) define goals that are monitored regularly and assessed with objective metrics; 4) develop a governance plan to ensure that the Research Agency remains under the full control of the ERS with a close relationship with the Science Council and, thus, includes ERS assemblies; 5) implement the plan with the help of fully dedicated experts and professionals; 6) promote activities where the ERS can add value and receive funding in the current research environment, both within and beyond the European union (EU); and 7) become financially self-sustaining and eventually expand its capacity.

It was also agreed that an ERS Research Agency should be clearly aligned with the strategic vision of the society and should provide means and opportunities for directly engaging ERS members in respiratory research not only at a pan-European level but also, given the new membership agreements, at a global scale. Finally, an ERS Research Agency would also closely align with the European Lung Foundation (ELF) involving patients at different levels in the research arena, while cooperating on strategic initiatives.

\section{Strategic areas of activity}

Based upon the core concepts outlined above, four strategic areas of activity were selected.

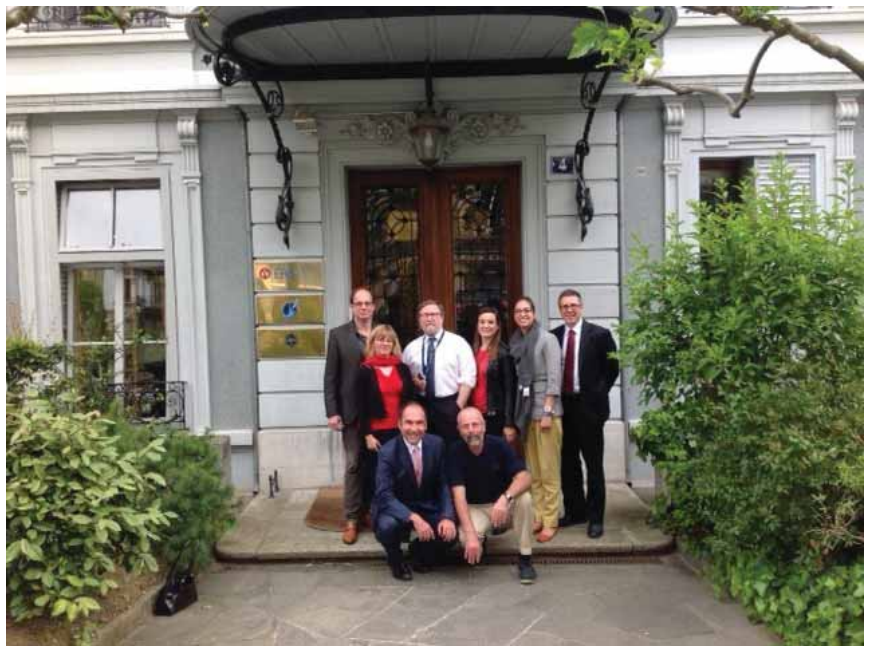

FIGURE 1 Initial core working group photo (Lausanne, May 22, 2014). In September 2015 the working group composition was redefined. 


\section{1) Standards, guidance and training}

The ERS excels in the provision of educational programmes and in bringing together the respiratory community through meetings, workshops, seminars and conferences, and the annual ERS International Congress. It is therefore well placed to help coordinate respiratory research by promoting collaboration, and to encourage best practice through the development and adoption of standards and by providing guidance and training.

As a first step, the activities of the ERS Research Agency could include: developing Europe-wide standard protocols and forms for data sharing and consent to data storage; developing education and training programmes on research methods, from experimental techniques to study design and statistics; and producing research guidelines and standard operating procedures (SOPs). This strategic activity would occur jointly with the existing Education pillar.

\section{2) Supporting researcher career development}

Fellowships are a successful aspect of the current ERS activities and essential elements of any research organisation. Furthermore, through its broad network of leaders in the respiratory field, the ERS represents an ideal coordinator for fellowship exchange programmes. Fellowships can also be an effective means to achieve strategic research objectives. The ERS Research Agency could help establish new fellowship programmes, could seek sponsors for fellowships and could develop partnerships with other funding bodies to increase the number of fellowships associated with respiratory research. The role of the Research Agency could be to identify the need for new programmes and to secure the necessary funding. Implementation and management of the Research Agency fellowships would still occur within the existing Science pillar.

\section{3) Obtaining funding, coordinating research projects and clinical studies}

The design and implementation of research studies could begin with ERS involvement in multinational grant-funded projects. As capacity develops, the ERS Research Agency would be well placed to coordinate and even manage large-scale pre-competitive clinical studies [7]. One of the big challenges is that clinical studies often use a variety of standards and protocols making study-to-study comparisons difficult. The ERS Research Agency could have an important role in driving a broader harmonisation of methods that facilitate and underpin larger clinical studies. This also represents an opportunity to encourage and promote the involvement of respiratory clinicians in clinical research, particularly from geographic regions that are underrepresented in current clinical studies. This effort need not be limited to clinical studies as the degree of harmonisation in the field of preclinical basic research is even less. Here, the Research Agency could assist in the design of research studies, and support the development of proposals for funding at the EU level by reviewing and approving studies, providing an "ERS seal of quality", and coordinating the conduct of multinational research projects and clinical studies.

As stated in the 5-year ERS strategic plan [6], it must be considered that, in time, the potential for facilitating and even conducting noncommercial drug clinical studies, or studies related to medical devices or clinical strategies to improve patient care, could all be possible within the Research Agency.

\section{4) Data}

There are a number of respiratory databases and registries either in existence (tuberculosis and bronchiectasis) or in development (pneumonia, chronic obstructive pulmonary disease and severe asthma) that are, and will be of value in the future for research. Substantial funding and effort by both researchers and patients was devoted to building these databases, and assessing their strengths and limitations is of the utmost relevance [8]. Assuring that the maximal value is derived from any given dataset is important [9].

ERS and ELF are currently taking part in a number of on-going projects (PRO-ACTIVE, U-BIOPRED, PREPARE and AirPROM) all of which are generating valuable datasets. Similar projects exist across many respiratory disease areas representing rich sources of data. There is also a growing interest in establishing patient registries and collecting more real life data. The ERS Research Agency could help to ensure that the maximal value is achieved from the data generated by: 1) funding the continuation of existing databases, registries, biobanks and sample collections; 2) providing additional services around the data, such as the ability to search across public and legacy datasets; 3) developing and running training on data handling and data analysis focused specifically around large datasets; 4) generating guidelines, SOPs and template consent forms for collecting and handling data in compliance with the EU legal and regulatory framework, and eventually establishing a central point to access datasets from multiple projects; and 5) supporting the further development of real life data registries and research networks. These are some of the areas where the Research Agency could immediately bring added value. Many of the challenges of building and/or hosting data centrally could be avoided by funding the continued hosting of data in its current location in return for making it available to the wider ERS community. It would not have to be available immediately 
or in its entirety. It could be that at the start access is limited and then expanded when the members of the project who generated the data are comfortable with wider access. Many projects have plans to publically release their data at some point in time. Consideration would also have to be given to ensuring that rules and regulations governing consent and data protection, both nationally and internationally, are followed.

\section{Structure}

ERS members will have essential roles within the ERS Research Agency, as participants in the oversight boards and as part of the process of developing new project ideas (figure 2). The Research Agency core team proposes that the main work of the agency would be divided into a number of programmes that are centered on a topic. Within each programme there would be a number of research tracks that would be responsible for the completion of specific projects. Each research track can include aspects related to the four strategic activities outlined above. Within each research track, a member lead for each strategic activity would be responsible for assuring the research track includes aspects relating to the respective strategic activity. A member chair would lead each research track and a number of at-large members would also be included, overall forming a member committee (figure 3). Each programme would be led by a research champion providing leadership for coordinating the research tracks and for developing sponsorships for new research tracks. Champions would be supported in the development of their programmes and research tracks by a Research Agency team from the ERS office and innovation project managers from a business development office (figure 4). In a stepwise manner, the business development office will also support efforts to obtain funding from grants, sponsors or fees for service projects along with the champions. The Research Agency office will provide logistical and administrative support. It is envisaged that staff from the office would eventually include information technology personnel, statisticians, bioinformaticians and data privacy experts as well as administrative staff. A board of trustees, which would include ERS leadership officers, would maintain oversight. The ERS would always maintain a voting majority in the board of trustees. A strategic advisory board comprised of external experts would be responsible for reviewing the performance of the Research Agency office head and the champions (figure 3). Its members would make recommendations regarding the renewal of the remit of the champions with the ERS Research Agency.

Access to data and knowledge (know-how) are assets the ERS Research Agency would develop and would be used as a central organising principle. Within the Research Agency office, a system would be maintained that allows members to access data that is under the control of the ERS Research Agency as well as public data. This would include both federated and centralised databases. Personal data protection regulations are likely to change soon in Europe and elsewhere, with new initiatives that aim to harmonise approaches to privacy and data protection [10]. However, this new EU legislation might threaten large scale research projects by trying to impose regulations for specific patient consent and reducing research exemptions. The Research Agency office would retain the services of a data protection expert. For the most part, the ERS Research Agency would limit the datasets it controls to those that are truly anonymised, but awareness of, and compliance with, the regulations may be essential for coordinating cohort studies where anonymisation is not possible [5]. In the USA, recent Federal initiatives aim to enhance protection of human research subjects, and improve its efficiency. These include: shortening and simplifying informed consent forms; categorising templates to enable the use of biological specimens for up to 10 years after collection; and approval to re-contact participants if secondary studies, registries, multicentre and ancillary studies should be facilitated [11]. Such measures are also likely to affect European-funded research.

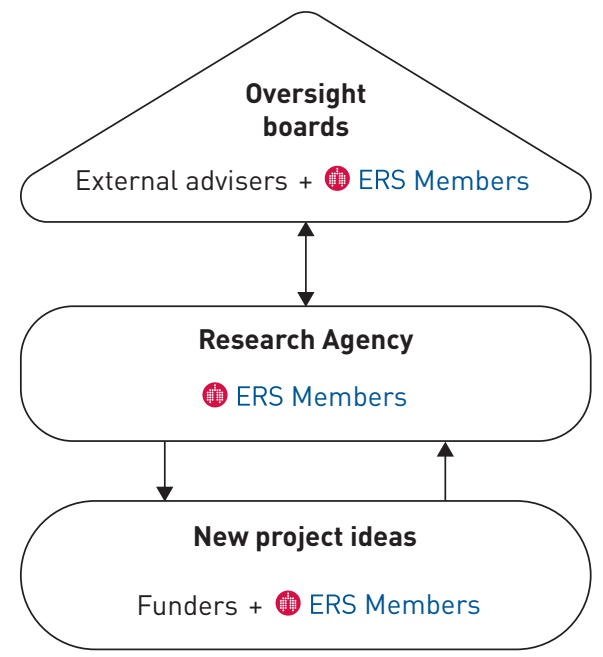

FIGURE 2 Overview of the European Respiratory Society (ERS) Research Agency structure. 


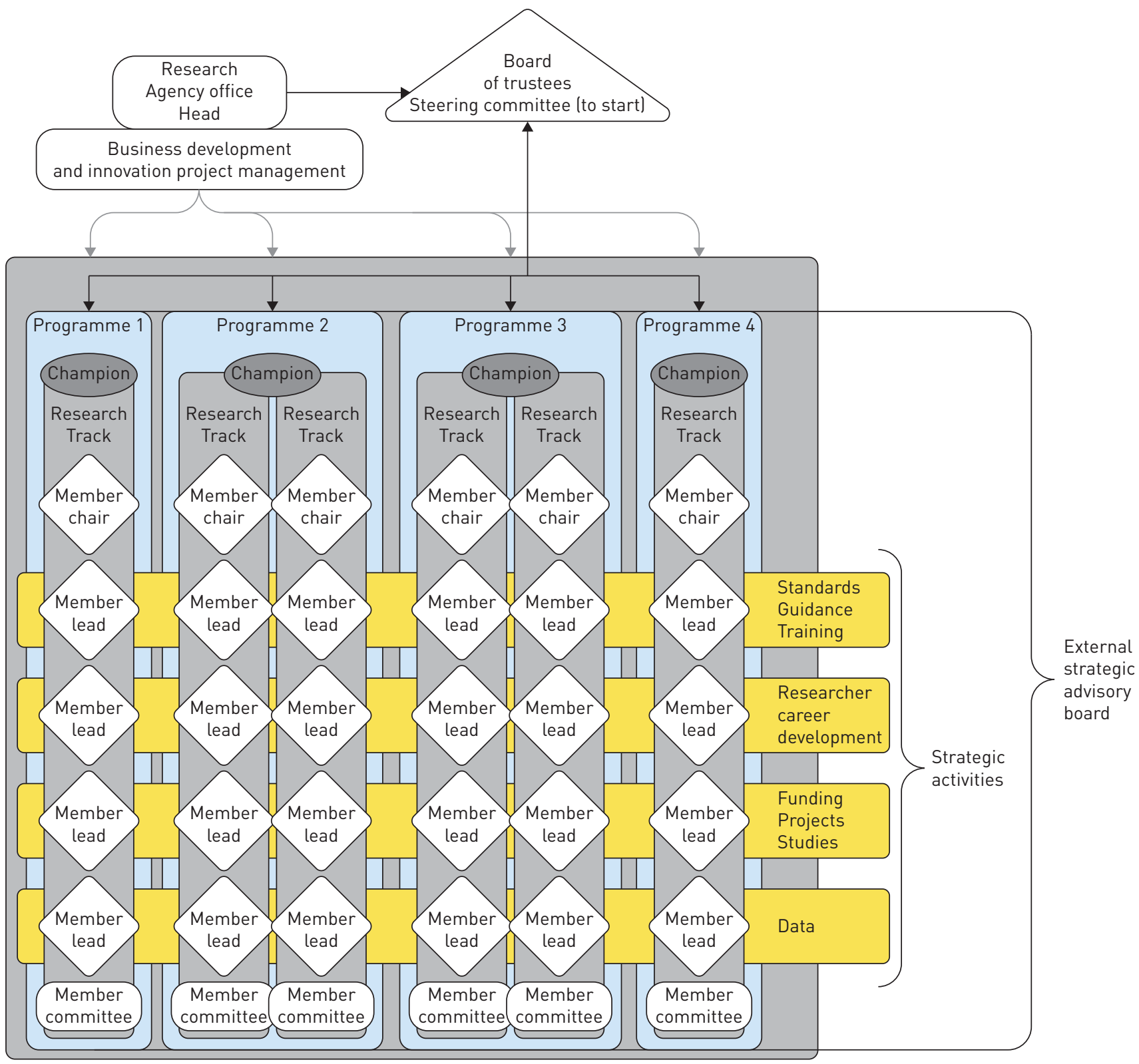

FIGURE 3 Proposed overall structure of the European Respiratory Society (ERS) Research Agency. Programmes comprised of multiple research tracks are supported and overseen by offices and boards.

The proposed structure is designed to be flexible and modular, and to combine both the breadth of expertise in the membership with the efficiency and drive of the Research Agency staff. Programmes and research tracks would be developed in a stepwise fashion. New research tracks would be formed and old research tracks decommissioned over time. The formation of research tracks is meant to be a way for the agency to respond as new research opportunities emerge.

ERS members, the champion and external funders would generate ideas for new research tracks. The Research Agency office and the business development office would support the champion(s) in forming ideas into plans for research tracks and projects. Champions would be judged on excellence, their ability to integrate ideas from various sources and to bring together research efforts in the fields relevant to their programme, among other performance measures [12].

\section{Sustaining and expanding the ERS Research Agency}

A financial model was developed internally to determine the potential viability of a Research Agency. This model, like any early stage model, contains a number of assumptions that will be tested and validated as the development of the Research Agency unfolds. The initiation of the Research Agency will be gradual, yet 


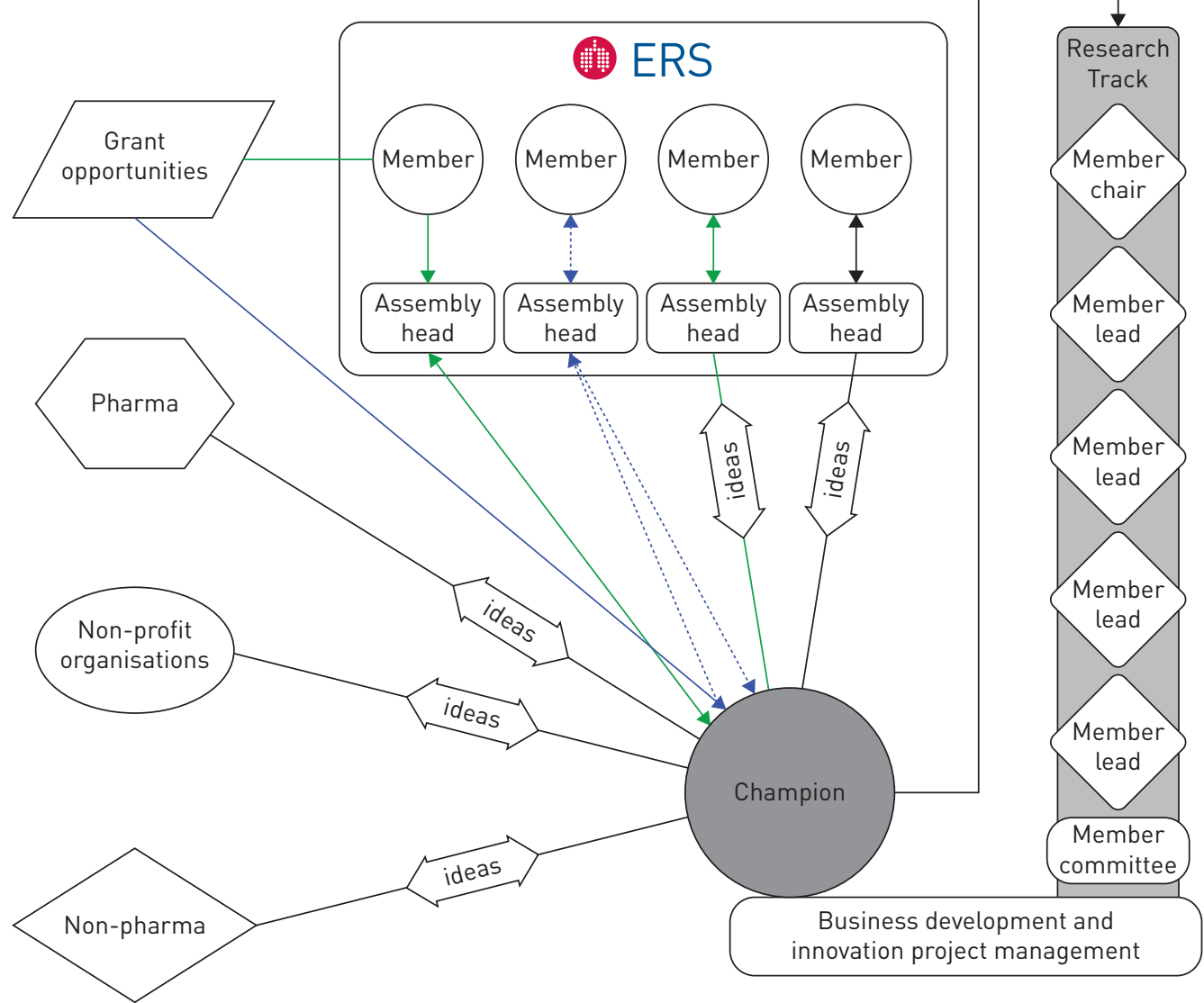

FIGURE 4 Development of ideas into research tracks and projects.

ambitious. At the outset an interim start-up team and governance structure would be established to take forward a number of initial steps designed to build capacity while realising some early successes. The initial "lean start-up" plan [13] includes: 1) understanding the research landscape, through at least two pilot programmes; 2) developing opportunities for funding; 3) piloting the concept of having champions; 4) investigating existing and establishing new registries; 5) setting up initial database infrastructure; and 6) exploring the development of educational programmes focused on research. This plan would take place over the course of a year, starting in September 2016, and allow for cautious validation of the planned expenditures and anticipated revenues.

\section{Summary}

There is at the current time a significant opportunity for the ERS to leverage its experience and reputation as an international umbrella organisation to promote high-quality, multinational respiratory research with the goal of improving the health of respiratory patients. This editorial proposes a model for the role and structure of an ERS Research Agency. It is based upon research, implicit knowledge and explicit feedback from ERS members and selected external individuals and organisations.

As with any new endeavour there are challenges and threats. Building a Research Agency will be a major undertaking that will require significant organisational planning, resources, effort and commitment. Organisations with multiple stakeholders tend to have a status quo inertia that has to be overcome for any significant new endeavour. The ERS Research Agency could be an investment in the future of respiratory research.

\section{Acknowledgements}

The Authors wish to thank the members of the ERS Management Group, Scientific Council and Executive Committee for their active participation and support to the project. Similarly, we acknowledge all the responses and constructive criticism from ERS members and allied organisations.

\section{References}

1 The burden of lung disease. In: Gibson J, Loddenkemper R, Sibille Y, et al., eds. The European Lung White Book. 2nd Edn. Sheffield, European Respiratory Society, 2013. www.erswhitebook.org/chapters/the-burden-of-lung-disease/ 
2 Vos T, Flaxman AD, Naghavi M, et al. Years lived with disability (YLDs) for 1160 sequelae of 289 diseases and injuries 1990-2010: a systematic analysis for the Global Burden of Disease Study 2010. Lancet 2013; 380: 2163-2196.

3 Snell N, Jarrold I, Holgate S. The current state of respiratory research in the UK. Thorax 2015; 70: 1011-1013.

4 Gross CP, Anderson GF, Powe NR. The relation between funding by the National Institutes of Health and the burden of disease. N Engl J Med 1999; 340: 1881-1887.

5 European Commission. Protection of personal data. European Data Protection Regulation, GDPR (2012/0011 COD) http://ec.europa.eu/justice/data-protection/ Date last accessed: April 16, 2015. Date last updated: Sept 17, 2015.

6 Migliori GB, Rabe KF, Bel E, et al. The European Respiratory Society plans its future: the 2013-2018 strategic plan. Eur Respir J 2014; 43: 927-932.

7 Holgate S, Agusti A, Strieter RM, et al. Drug development for airway diseases: looking forward. Nat Rev Drug Discov 2015; 14: 367-368.

8 von Ungern-Sternberg BS, Regli A. Big problem, small incidence, and large registry datasets. Lancet Respir Med 2016; 4: 5-6.

$9 \quad$ Drazen JM. Sharing individual patient data from clinical trials. N Engl J Med 2015; 372: 201-202.

10 The Lancet Respiratory Medicine. Data protection: balancing personal privacy and public health. Lancet Respir Med 2016; $4: 1$.

11 Emanuel EJ. Reform of clinical research regulations, finally. N Engl J Med 2015; 373: 2296-2299.

12 Grether M, Eickelberg O, Mall MA, et al. New metrics for translational research. Lancet Respir Med 2014; 2: e13-e14.

13 Ries E. The Lean Startup: How Today's Entrepreneurs Use Continuous Innovation to Create Radically Successful Businesses. New York, Crown Publishing, 2011; p. 103. 